\title{
MEASUREMENT SYSTEM WITH HALL AND A FOUR POINT PROBES FOR CHARACTERIZATION OF SEMICONDUCTORS
}

\author{
Rudolf Kinder* — Miroslav Mikolášek * Daniel Donoval * \\ Jaroslav Kováč ${ }^{*}$ - Marek Tlaczala ${ }^{* *}$
}

\begin{abstract}
This article describes the design, construction and control program of an automatic measuring system (AMS), which was designed for sheet resistance and sheet Hall mobility measurements. The van der Pauw technique and 4-point probe method are used to measure sheet resistance and calculate the specific resistance. Furthermore, the software of the AMS contains graphical subroutines for conversion of resistivity to concentration and mobility. Hall effect and electronic transport measurements are invaluable for understanding and characterization of the physical properties of semiconductors such as $\mathrm{Si}$, GaAs, $\mathrm{ZnO}$, and nanocrystal diamond as well as other electronic and magnetic materials.
\end{abstract}

K e y w o r d s: Hall system, van der Pauw, 4-point probe, Si, ZnO

\section{INTRODUCTION}

An important part in production of electronic components and integrated circuits is the diagnostics of basic material parameters. The van der Pauw method is one of the most utilized measurement methods for evaluation of the semiconductor material electrical properties, such as resistivity, carrier density, and mobility [1]. Method can be used to measure samples of arbitrary shape, although several basic sample conditions must be satisfied to obtain accurate measurements. Among them the most important are good homogeneity of the sample, constant thickness of the sample and in ideal case infinitive small point contacts placed at the edges of the samples [2]. Moreover, the quality of contact in terms of good ohmic behavior has to be assured. The van der Pauw method is a common method used for investigation of new prepared thin films to determine their quality. Characterizing the mobility of new materials is essential for semiconductor technology innovations, so making accurate Hall effect measurements will continue to be important [2].

Additional resistivity measurement method, the 4point probe, is also widely used in the semiconductor industry to monitor the production process. Electrical measurements are done on test structures to provide information on the various process steps. For example, resistivity measurements of doped semiconductor structures provide information about the carrier concentration and mobility and are used as feedback for the doping diffusion process [3].

This paper describes an AMS with Hall and the 4point-probe method. Optimum measurement conditions for determination of mobility $\mu$ are established for different semiconductor layers and structures. Hall effect is invaluable to understand and characterize the physical properties of semiconductors including materials such as:
Si, Ge, GaAs, AlGaAs, ZnO, and others. Transport measurements of Hall effect and magnetoresistance are ideally suited for material research, product development, and quality control $[2,45]$.

\section{THEORY}

The designed automatic measuring system can be used to determine several material parameters: the Hall voltage $\left(V_{\mathrm{H}}\right)$, carrier concentration $(n)$, Hall coefficient $\left(R_{\mathrm{H}}\right)$ and the conductivity type ( $n$ or $p$ ) are all derived from the Hall voltage measurement. To be able to evaluate carrier mobility $(\mu)$ it is necessary to measure also the resistivity of the sample $(\rho)$. Due to the required contact node arrangement shown in Fig. 1, the van der Pauw method is the most frequently used method in the Hall measurement systems to evaluate the sample resistivity.

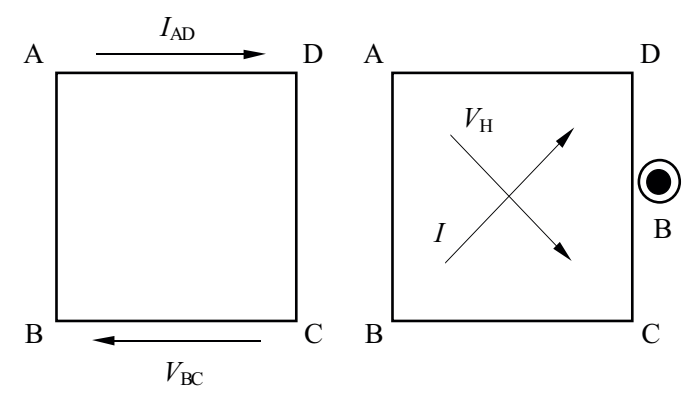

Fig. 1. Contact node arrangement for the van der Pauw and Hall measurement

For Hall measurements a contact configuration gives current and voltage perpendicular to each other. Resistivity measurement configurations $\mathrm{R}_{(\mathrm{A}, \mathrm{C} / \mathrm{B}, \mathrm{D})}$ or $\mathrm{R}_{(\mathrm{B}, \mathrm{D} / \mathrm{A}, \mathrm{C})}$ are possible and can be selected for the measurement. For ideal square symmetry samples the measured voltage at zero magnetic field should be 0 independently of the used current [1-4].

\footnotetext{
* Slovak Technical University, Faculty of Electrical Engineering and Information Technology, Institute of Electronics and Photonics, SK-812 19 Bratislava, rudolf.kinder@stuba.sk; ${ }^{* *}$ Institute of Microsystems Technology, Wroclaw University of Technology, Faculty of Electronics, Janiszewskiego 11/17, 50372 Wroclaw, Poland
} 
For Hall effect measurements, a voltage is applied between the contacts placed at diagonally opposite corners and the current $I$ flowing between them is measured. In addition, a magnetic field $B$ is applied in the direction perpendicular to the sample and the change in Hall voltage $V_{\mathrm{H}}$ between the contacts in opposite corners is measured. Resistivity $\rho$, carrier density $n$, and mobility $\mu$ are calculated from the measured values, applied magnetic field $B$ and the film thickness $d$ of the measured sample [2].

According to $[1,4]$ the van der Pauw method consists of two measurements of resistivity, $R_{\mathrm{A}}$ and $R_{\mathrm{B}}$

$$
\begin{aligned}
& R_{\mathrm{A}}=\left(R_{\mathrm{DA}, \mathrm{CB}}+R_{\mathrm{AD}, \mathrm{BC}}+R_{\mathrm{BC}, \mathrm{AD}}+R_{\mathrm{CB}, \mathrm{DA}}\right) / 4, \\
& R_{\mathrm{B}}=\left(R_{\mathrm{CB}, \mathrm{BA}}+R_{\mathrm{DC}, \mathrm{AB}}+R_{\mathrm{AB}, \mathrm{DC}}+R_{\mathrm{BA}, \mathrm{CD}}\right) / 4 .
\end{aligned}
$$

From these two measurements and the knowledge of the sample thickness under test $d$, the conductivity of arbitrary shaped samples can be determined by the solution of the following equation

$$
q\left(-\pi R_{\mathrm{A}} / R_{S}\right)+q\left(-\pi R_{\mathrm{B}} / R_{S}\right)=1 .
$$

To simplify the solution, it is appropriate to measure square shaped samples, where $R_{\mathrm{A}} \approx R_{\mathrm{B}}$. In this case the solution of Eq. (2) can be written as [2]

$$
\rho=R_{S} d=\frac{\pi d}{\ln 2} \frac{\left(R_{\mathrm{AB}, \mathrm{CD}}+R_{\mathrm{BC}, \mathrm{DA}}\right)}{2} f
$$

where

$$
R_{\mathrm{AB}, \mathrm{CD}}=\frac{V_{\mathrm{CD}}}{I_{\mathrm{AB}}}, \quad R_{\mathrm{BC}, \mathrm{DA}}=\frac{V_{\mathrm{DA}}}{I_{\mathrm{BC}}}
$$

and $f$ represents the correction factor, which is function of sample symmetry. Within AMS system, Equation (2) is solved numerically using PC software, so correction factor $f$ has only an informative value. From the Hall voltage measurements it is possible to determine Hall coefficient using equation

$$
R_{\mathrm{H}}=\frac{V_{\mathrm{H}} d}{I B}
$$

and consequently carrier concentration can be determined

$$
n=\frac{1}{R_{\mathrm{H} q}}
$$

where $q$ is the elementary charge. Finally, Hall coefficient and resistivity are used to determine hall mobility

$$
\mu=\frac{R_{\mathrm{H}}}{\rho} .
$$

To provide a good accuracy of obtained parameters it is necessary to ensure some basic conditions during the measurement. The most crucial one is to have good ohmic contacts. The van der Pauw theory requires infinitive small contacts in the corner of the sample [1]. Very important is also to ensure negligible heating of the sample during the measurements, sensitive current measurement, and good symmetry and homogeneity of the sample.

The 4-point probe technique is one of the most common experimental methods for measuring resistivity. This configuration consists of four independent electrical terminals where two terminals are used to apply current to the sample, and the other two terminals measure the resulting potential drop across the defined portion of the sample $[3,5]$. In the technique of linear 4 -point probes all measurement tips are placed along a straight line and separated by a constant distance. The resistivity $\rho$ of a homogeneously doped semiconductor can be then calculated as

$$
\rho=R_{s} d=4.53 \frac{V}{I} d k,
$$

where $d$ is the semiconductor sample thickness, $I$ is the current flowing through the outer tips of the probe, $V$ is the voltage drop measured on the inner tips, $k$ is the correction factor. The accuracy of calculating the parameters by the linear 4-point probe is described elsewhere [5].

The concentration values $n$ can be obtained from the measured values $\rho$ using the conversion graphs, so-called Irvin curves for $n$ - and p-type. Irvin curves express the mutual dependence between resistivity $\rho$, mobility $\mu$ and concentration $n$ according to the formula

$$
n(x)=\frac{1}{\rho(x) \mu(x) q} .
$$

For automatic calculation of $\rho$ or $n$, the value of $\mu$ is used given by empirical formula

$$
\mu=\mu_{\min }+\frac{\mu_{\max }-\mu_{\min }}{\left(1+\frac{n}{n_{\mathrm{ref}}}\right)^{\alpha}} .
$$

Here $\mu_{\min }$ and $\mu_{\max }$ are the values of minimal and maximal mobility; $n_{\text {ref }}$ is the reference concentration and $\alpha$ is an exponential factor [5].

\section{DESIGN OF EQUIPMENT AND MEASUREMENT ROUTINE}

The measurements and calculations were carried out on automatic measuring system (AMS) designed at the DSLab. s.r.o. in collaboration with Institute of Electronics and Photonics, STU in Bratislava. The AMS provides measurements of sheet resistivity $R_{S}$ and Hall voltage $V_{\mathrm{H}}$, to evaluate specific resistivity $\rho$, carrier concentration $n$, Hall mobility $\mu$ and type of semiconductor (ntype or p-type). Two different ways were used to measure resistivity: (i) Van der Pauw set-up being the part of the Hall measurement covering the sheet resistance in the range from 0.1 to $10^{10} \Omega / \mathrm{cm}^{2}$ and (ii) linear 4-point probe providing measurements in the range of specific resistivity from $10^{-3}$ to $20 \Omega \mathrm{cm}$. A part of measuring system is the sample board allowing measurements at 


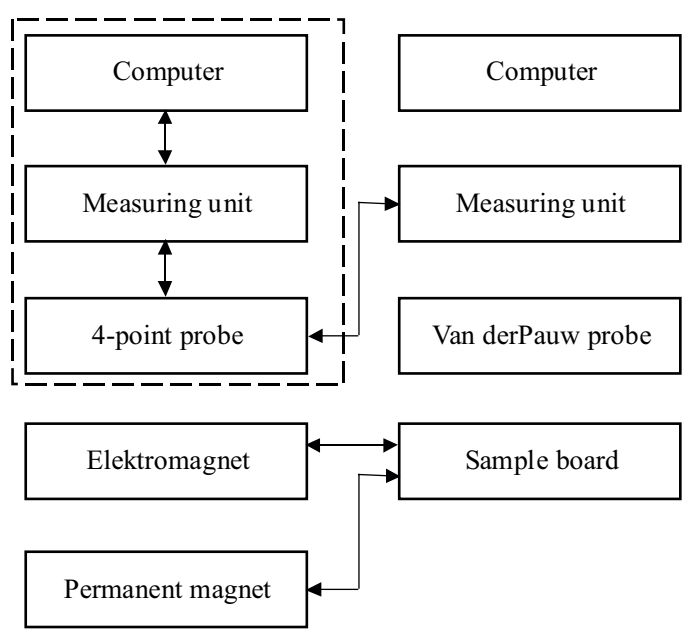

Fig. 2. The complete automatic measuring system

room and liquid nitrogen temperature utilizing permanent magnet $(0.55 \mathrm{~T})[6]$ or electromagnet $(0.15 \mathrm{~T})$. The sample is electrically contacted through Ecopia corporation sample holder [6]. The block diagram of complete measuring system is shown in Fig. 2.

The conversion of $\rho$ to $n$ for $\mathrm{p}$ - or n-type Si according to Eqs. (9) and (10) can be performed by a conversion code. For determining $R_{\mathrm{S}}, \rho$ and $n$ it is needed to know following parameters: type of semiconductor, thickness of sample or layer, correction factor value, given current (max. $100 \mathrm{~mA}$ ) and number of measurements. Then from selected number of measurements the average values of $R_{\mathrm{s}}, \rho$ and $n$ are calculated (Eqs. (8), (9), (10)) [5].

The value of sheet resistance required for evaluation of Hall mobility and concentration is measured using van der Pauw configuration. Various aspects are important to obtain a good sensitivity and accuracy using this method. The most critical role in the measurements play the contacts created on the samples [7]. The quality of ohmic contacts in the van der Pauw configuration is controlled via the measurements of I-V linearity between neighboring contacts, where four leads labeled as A, B, C, and D (Fig. 1) are connected to the four contacts on the edges of the sample. The technology of ohmic contacts formation differs for various semiconductor samples and is described elsewhere [7].

The sample of good homogeneity and symmetry is required for accurate resistivity measurements. These two sample quantities are inspected through the resistivity measurement, $R_{\mathrm{A}}$ and $R_{\mathrm{B}}$, carried on two sides of the square sample. $R_{\mathrm{A}}$ divided by $R_{\mathrm{B}}$ then gives the information on sample symmetry and should have the value in the range of $0.9-1$.

Another aspect influencing the measurement accuracy, power dissipation during the measurements is automatically controlled by software as well. Power dissipation in designed measurement system is controlled and a warning signal is generated when a prescribed value (in $\mathrm{mW}$ ) is overcome.
The Hall system together with the van der Pauw resistivity configuration was primary designed for high resistance sample measurements. For this kind of measurements, the voltage source was used to feed the sample, so the current through sample is limited only by semiconductor resistivity. The current measurements are provided with currents ranging from $100 \mathrm{pA}$ to $10 \mathrm{~mA}$ with the highest sensitivity of $0.02 \mathrm{pA}$.

For Hall measurements a contact configuration perpendicular to each other is expected for current flow and voltage measurement. For ideal samples the measured voltage at zero magnetic field should be zero independently of the used current. In a real sample, due to nonperpendicular contact misalignment, the voltage offset is measured also at zero magnetic field, which present a serious problem for measurements of low Hall voltage. In some cases, if this misalignment voltage is much higher than the Hall voltage, this voltage can cause a big systematic error. To overcome this problem, Hall system contains a hardware compensation of misalignment voltage and gives much better results on asymmetric samples as Hall systems without it. The compensation mode can be switched on by software. The range of Hall voltage measurements goes from $5 \mathrm{mV}$ to $10 \mathrm{~V}$ with the highest sensitivity $0.05 \mu \mathrm{V}$ provided at the lowest voltage range.

The measuring system is fully automated. The menu driven software of AMS insures communication with the measuring equipment, commands its single parts, measurement of the required parameters and communication with the operating personnel. The operating personnel by means of the driven software enters the data needed for the measurement process and at the same time the results of measurement form a listing on the display or a file. As development tools, the operating systems MS Windows and Borland C Builder 6 were used. The control graphical interface of AMS for Hall, 4-point probe and van der Pauw measurements is shown in Fig. 3.

As can be seen on the left hand side of the front panel shown in Fig. 3, main parameters to be set before measurements are sample thickness, initial voltage, and number of measured points of I-V curves for contacts linearity control. For resistivity and Hall measurements, it is necessary to set the value of initial approximate current used during the sample check and number of Hall voltage averaging together with Hall voltage measurement range. In this part of the panel, it is also possible to set optional linear 4-point probe measurements or Hall measurements with included van der Pauw resistivity measurements. The middle part of the control graphical interface gives to user basic information about voltages and current at particular nodes of the sample and information about compensation voltage at zero magnetic field and measured Hall voltage. Measured values of Hall voltage, resistivity and parameters determined from these values are set in the right part of the control graphical interface.

The 4-point probe measurement of resistivity is controlled by separate computer. The control software allows setting the optional linear or square 4-point probe arrangement, conductivity type of the semiconductor, film 


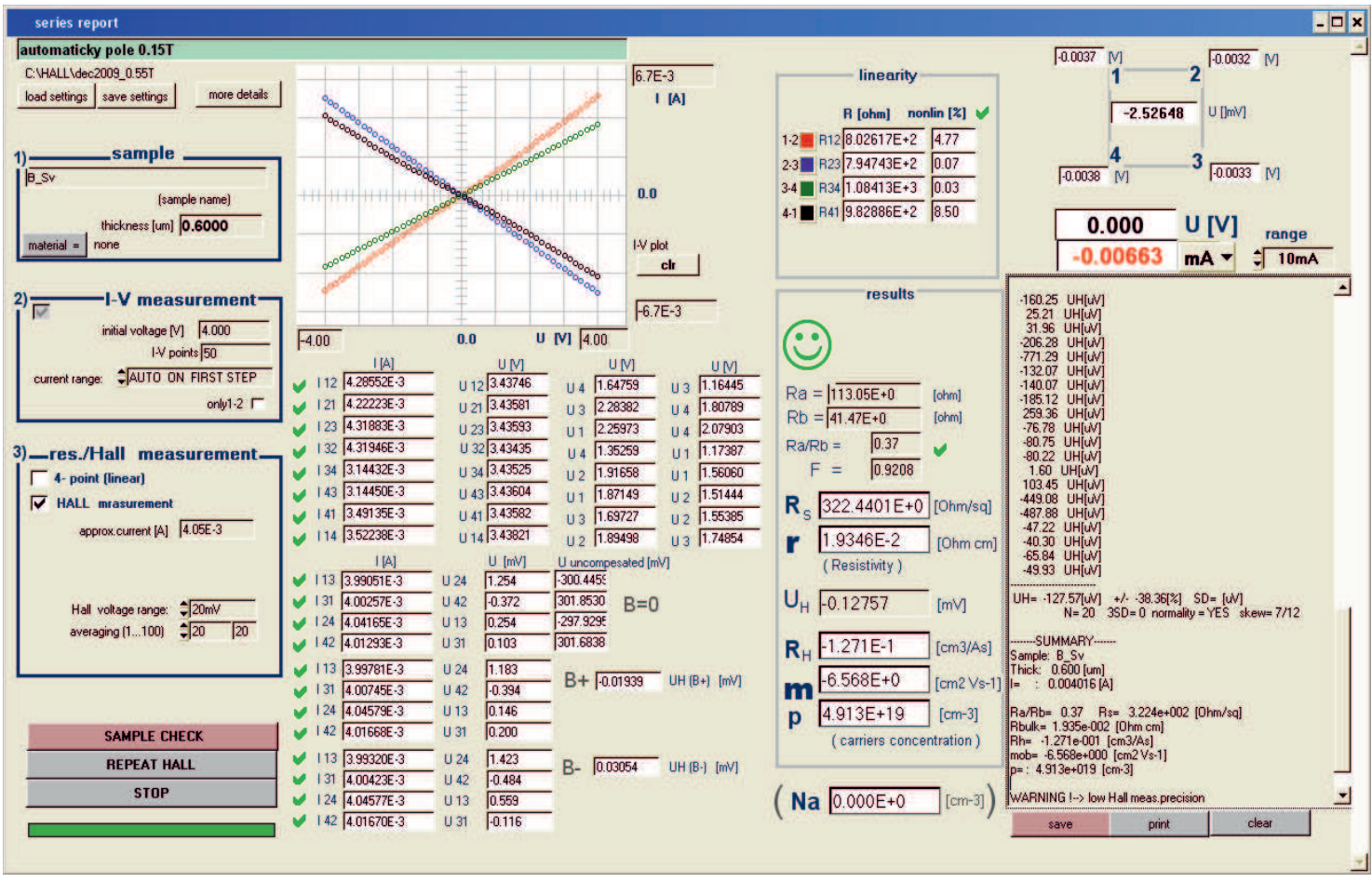

Fig. 3. Control graphical interface of AMS for Hall, linear 4-point probe and van der Pauw measurements

thickens $d$, correction factor, current and number of the measurements. The measured and evaluated values are spreading resistance, resistance and concentration.

\section{EXPERIMENTAL RESULTS}

Designed AMS system allows measuring and calculating various parameters such as resistivity, Hall mobility and doping concentration. To demonstrate applicability and reliability of this system, we conducted various experiments described in details below.

To verify the resistivity measurements provided by linear 4-point probe being part of the AMS, two wafers were used: p-type $\mathrm{Si}\langle 100\rangle$ substrate with $\rho=8-12 \Omega \mathrm{cm}$ and thickness of $300 \mu \mathrm{m}$ and n-type $\mathrm{Si}\langle 100\rangle$ substrate with $\rho=2-4 \Omega \mathrm{cm}$ and thickness of $360 \mu \mathrm{m}$. The values of $\rho, n$ and $\mu$ were measured and calculated by using AMS. For both samples, the value of $k$ was 0.9818 (Eq. 8). For the p-type Si sample we measured values: $R_{s}=345 \Omega, \rho=10.3 \Omega \mathrm{cm}, n=1.2 \times 10^{15} \Omega \mathrm{cm}^{-3}$, $\mu=485 \mathrm{~cm}^{2} / \mathrm{Vs}$. For the n-type $\mathrm{Si}: R_{s}=108.6 \Omega$, $\rho=3.3 \Omega \mathrm{cm}, n=4 \times 10^{15} \mathrm{~cm}^{-3}, \mu=472 \mathrm{~cm}^{2} / \mathrm{Vs}$. The above-mentioned results $\rho$ and $n$ satisfy the sample technological parameters.

For the verification of $\rho$ to $n$ conversion p-type silicon substrate with $\rho=10 \Omega \mathrm{cm}$ and n-type silicon substrate with $\rho=1 \Omega \mathrm{cm}$ were used. Obtained results of conversion are for p-type Si: $n=1.29 \times 10^{15} \mathrm{~cm}^{-3}, \mu=485 \mathrm{~cm}^{2} / \mathrm{Vs}$ and for n-type Si: $n=5.3 \times 10^{15} \mathrm{~cm}^{-3}, \mu=1180 \mathrm{~cm}^{2} / \mathrm{Vs}$. The calculated results are identical with the results obtained from Irvin curves [5].
To demonstrate the potential of Hall measurement tool in current most attractive topics in material research, measurements on four kinds of semiconductor samples with different resistance were carried out:

- sample c-Si(p): High doped p-type crystalline silicon substrate with thickness of $450 \mu \mathrm{m}$. Silicon is still most used material in semiconductor industry and part of various structures and devices.

- sample $\mathrm{ZnO}: \mathrm{Al}: 1.1 \mu \mathrm{m}$ thick aluminum doped zinc oxide layer prepared on glass substrate. $\mathrm{ZnO}$ is a unique material and has been used considerably for its catalytic, electrical, optoelectronic and photochemical properties.

- sample GaAs:Si: Si doped GaAs epitaxial layer of thickness $1.25 \mu \mathrm{m}$ grown by molecular beam epitaxy on GaAs-SI (semi-insulating) substrate. GaAs based heterostructures have the advantage for realization of micro and optoelectronic devices.

- sample Diamond: nanocrystalline diamond layer of $0.9 \mu \mathrm{m}$ thickness deposited on $1.4 \mu \mathrm{m}$ thick $\mathrm{SiO}_{2}$ insulating layer on silicon substrate. To overcome the leakage current caused by diamond layer, which during the deposition overlaps over the thin insulating $\mathrm{SiO}_{2}$ layer and makes connection to the silicon substrate, the edges of the diamond layer were etched. Diamond is a robust semiconductor with a wide bandgap of $5.48 \mathrm{eV}$, and it could potentially be used in applications that need to function in environments of extreme heat and radiation.

- sample BDiam: boron doped diamond layer of thickness $0.2 \mu \mathrm{m}$ deposited on $1.4 \mu \mathrm{m}$ thick $\mathrm{SiO}_{2}$ insulating layer on silicon substrate. The edge of the sample was 


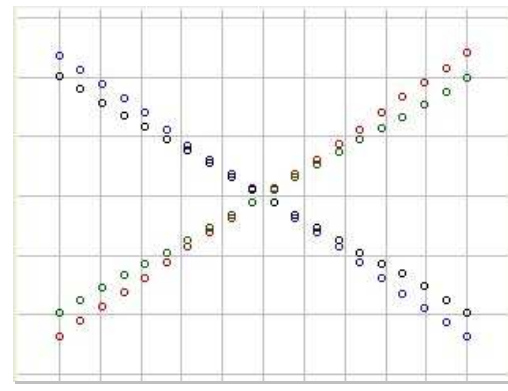

$\mathrm{ZnO}: \mathrm{Al}$

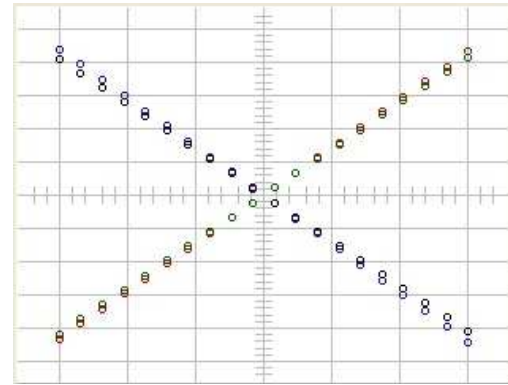

BDiam

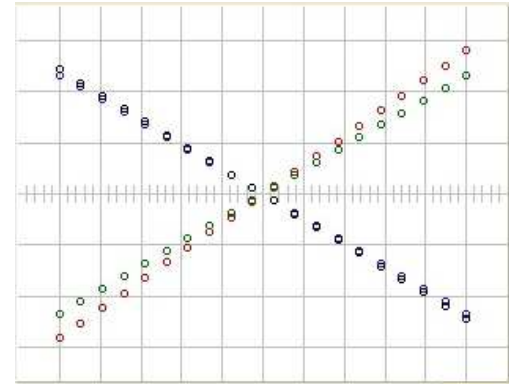

C-Si(p)

Fig. 4. Contact linearity of measured samples: $\mathrm{ZnO}: \mathrm{Al}, \mathrm{BDiam}$ and c-Si(p)

Table 1. Summarized results of Hall measurements. In the table $d$ is sample thickness, $R_{s}$ is sheet resistivity, $\rho$ is specific resistivity, $U_{\mathrm{H}}$ is Hall voltage, $\mu$ is mobility and $n$ is carrier concentration

\begin{tabular}{cccccccc}
\hline Sample & $\begin{array}{c}d \\
(\mu \mathrm{m})\end{array}$ & $\begin{array}{c}R_{s} \\
\left(\Omega / \mathrm{cm}^{2}\right)\end{array}$ & $\begin{array}{c}\rho \\
\text { Omega cm }\end{array}$ & $\begin{array}{c}\text { Contact } \\
\text { nonlin. } \%\end{array}$ & $\begin{array}{c}U_{\mathrm{H}} \\
(\mu \mathrm{V})\end{array}$ & $\begin{array}{c}\mu \\
\left(\mathrm{cm}^{2} \mathrm{Vs}^{-1}\right)\end{array}$ & $\begin{array}{c}n \\
\left(\mathrm{~cm}^{-3}\right)\end{array}$ \\
\hline c-Si(p) & 450 & 0.0438 & $1.97 \times 10^{-3}$ & $<1$ & 0.25 & 49.2 & $6.4 \times 10^{19}$ \\
ZnO:Al & 0.750 & $4.587 \times 10^{1}$ & $3.4 \times 10^{-3}$ & $<8.01$ & 79.40 & 10.2 & $8 \times 10^{20}$ \\
GaAs:Si & 1.25 & $6.24 \times 10^{2}$ & $7.8 \times 10^{-2}$ & $<0.2$ & 33330 & $3.5 \times 10^{3}$ & $2.2 \times 10^{16}$ \\
Diamond & 0.9 & $3.6 \times 10^{7}$ & $3.3 \times 10^{3}$ & $<10$ & 130 & 1.5 & $1.2 \times 10^{15}$ \\
BDiam & 0.2 & $2.2 \times 10^{3}$ & $4.4 \times 10^{-2}$ & $<1$ & 45 & 1.3 & $1.1 \times 10^{20}$ \\
\hline
\end{tabular}

cut in order to prevent the leakage via the silicon substrate. Doped diamond can be used as a core material of sensors, MEMS and MESFET.

In the first step of measurements, the samples were attached to the holder and four contact labels were placed on the corners of the sample. The quality of the ohmic contacts to the sample was controlled via the resistance linearity measurement between neighboring contacts. In case of the $\mathrm{ZnO}: \mathrm{Al}$ sample, which has a high conductance, the good ohmic contact giving nonlinearity value bellow $0.5 \%$ was attained by direct placing of sample holder gold tips on the sample. Other samples required contact preparation. For samples with diamond layer and GaAs:Si layers an In contacts were first spread at the corners of the samples and than annealed in FGA $\left(90 \% \mathrm{~N}_{2}+\right.$ $\left.10 \% \mathrm{H}_{2}\right)$ at the temperature of $400{ }^{\circ} \mathrm{C}$ for 1.5 minutes. The aluminum contacts were evaporated on the corners of c-Si(p) substrate to assure ohmic contact.

As is shown in the Fig. 4, sufficient linearity of I-V curves between neighbored contacts and thus good ohmic contacts were achieved for all samples. The results of resistivity and Hall measurements for selected samples are summarized in Tab. 1. All Hall measurements were conducted by averaging over 20 measurements to improve the statistics. Such a high number of measurements were provided using electromagnet.

Measurements of resistivity provide information in development of new materials for various applications. Zinc oxide represents attractive alternative of transparent conductive oxide (TCO) for solar cell applications. Resistivity of TCO is one of the most important parameters significantly affecting the performance of solar cells, thus need to be controlled. The obtained resistivity of $\mathrm{ZnO}$ noticed in Tab. 1. [8] is usually achieved in case of state of the art $\mathrm{ZnO}$ layers for TCO applications in solar cells.
Diamond is an attractive material for sensors, MEMS and MESFET transistors $[9,10]$. Important feature for such applications is a good conductivity of diamond layers to achieve ohmic contact. Low resistance ohmic contact can be achieved for layers when the current crosses a very thin potential barrier between the metal and the diamond. While the barrier thickness decreases upon the increase of dopant concentration, the Hall measurements are important to optimize the ohmic contacts [11]. Results of resistivity and Hall measurements obtained on intrinsic and boron doped diamond films (Tab. 1) represent the basic steps in the process of ohmic contact optimization.

Obtained results are in good agreement with technological predictions and measurements carried out on other Hall measurement tools [8]. The developed system proved the potential of reliable resistivity, mobility and doping concentration measurements on various semiconductors.

\section{CONCLUSION}

The classical Hall measurement system has been completed by a 4-point probe method, which employs a relay network for measuring the sheet resistance and then calculates the resistivity of semiconductor layers. The Hall system together with the van der Pauw resistivity configuration was primary designed for high resistance sample measurements. Two different ways were used to measure resistivity (i) Van der Pauw set-up being the part of the Hall and (ii) 4-point linear probe. The software of the equipment contains graphics for converting the resistivity into concentration and mobility.

The measuring system allows measurements utilizing permanent magnet $(0.55 \mathrm{~T})$ or electromagnet $(0.15 \mathrm{~T})$ and is suitable for determination of the influence the disturbances on the measured mobility. Special feature 
of the equipment is generation of magnetic field, which is realized with air-cooled magnet with fast switching magnetic pole $0.15 \mathrm{~T}$. This has the advantage to minimize an excessive heat generation in some samples and also exhibit lower false thermovoltages in sample contacts. The quickly switch combination are especially advantage for a sample with low Hall voltage (less than $50 \mu \mathrm{V}$ ).

The potential of developed tool was attested by measurement on various types of semiconductor samples and structures. Potential problems inherent to the AMS have been described and minimized as far as possible.

\section{Acknowledgement}

The work has been conducted in the Centre of Excellence CENAMOST supported by the grants VEGA 1/0377/13 and Project of International Research Collaboration, SKPL-0005-12: TU Wroclaw/STU Bratislava. The authors would like to thank Vladimír Kremničan, director of DSLab. s.r.o. company for design and manufacture of this equipments.

\section{REFERENCES}

[1] VAN DER PAUW, L. J.: A Method of Measuring Specific Resistivity and Hall Effect of Discs of Arbitrary Shape, Philips Research Reports, 13, 1958, 1.

[2] MAtSumuRA, T.-SATO, Y.: A Theoretical Study on Van Der Pauw Measurement Values of Inhomogeneous Compound Semiconductor Thin Films, J. Mod. Phys. 1 (2010), 340.

[3] SChuetze, A. P. et al: Am. J. Phys. vol 72 No. 2 (Feb 2004), 49.

[4] GREEN, R. : Hall Effect Measurements in Materials Characterization, Keithley Instruments, Inc., Keithley Instruments, Inc. $2 \mathrm{O} 11$.

[5] KINDER, R. et al : Journal of Electrical Engineering 56 (2005), 268-270.

[6] http://four-point-probes.com/ecopia.html.

[7] www.ecse.rpi.edu/ schubert/.../ A07-Specific-contact-resistance.pdf.

[8] FLICKYNGEROVÁ, S.-NETRVALOVÁ, M.-ŠUTTA, P.NOVOTNY, I.-TVAROŽEK, V.-GAŠPIERIK, P.-BRUNCKO, J. : Effects of Sputtering Power and Pressure on Properties of ZnO:Ga Thin Films Prepared by Oblique-Angle Deposition, Thin Solid Films 520 No. 4 (2011), 1233.

[9] MARTON, M.-IŽÁK, T.-VESELÝ, M.-VOJS, M.-MICHALKA, M.-BRUNCKO, J. : Effect of Argon and Substrate Bias on Diamond Thin Film Surface Morphology, Vacuum. 82 (2008), 154.

[10] IŽÁK, T.-VOJS, M.-VESELÝ, M.-ŠKRINIAROVÁ, J.NOVOTNÝ, I.-MICHALKA, M.-REDHAMMER, R. : Electrical Property Dependence on Thickness and Morphology of Nanocrystalline Diamond Thin Films, Microelectronics Journal 40 (2009), 615

[11] WERNER, M.-DORSCH, O.-BAERWIND, H. U.-ERSOY, A.-OBERMEIER, E.-JOHNSTON, C.: Diamond Relat. Mater. 3 (1994), 983.

Received 16 September 2012

Rudolf Kinder (doc, Ing, CSc) was born in Pezinok in 1940. In the sixties, he worked in electrotechnical industry in Bratislava, later at VURUP (Research Center for Petrol Chemistry) in the field of automation of chemical services. He graduated in technical cybernetics from the Faculty of Electrical Engineering, Slovak Technical University, Bratislava. He received $\mathrm{CSc}(\mathrm{PhD})$ degree in electronics and vacuum technology in 1984 and has worked as a senior scientist since 1989. Since 1996 he has been Associate Professor in microelectronics. His research activities are oriented towards semiconductor technology, particularly to analysis and simulation.

Miroslav Mikolášek (Ing), (Ing, PhD.) was born in Myjava (Slovakia), in 1983. He carried out research in the area of microfluidic circuits at the Johannes Kepler University in Linz, Austria, and based on this work he received his Master degree in electronics from the Slovak University of Technology, Bratislava, in 2007. In year 2011 he recived $\mathrm{PhD}$ degree in Electronics from the Slovak University of Technology. At present he works at the Institute of Electronics and Photonics, Slovak Technical University in Bratislava. Main interests of his research include simulation and diagnostics in the field of solar cells based on amorphous silicon.

Daniel Donoval (Prof, Ing, DrSc) was born in Banská Bystrica, Slovakia in 1953. He received his MSc and PhD degrees in electronics from Slovak University of Technology in 1976 and 1981, respectively. Since 1981 he has been with Microelectronics Department, FEI STU Bratislava. He he is currently a Professor and Head of the Department of Electronics and Phototonics. His research interests include technology and characterization of semiconductor structures and devices supported by $2 / 3-\mathrm{D}$ modeling and simulation. He is a member of Scientific Community Council and Education and Training Coordination Board of European Technology Platform ENIAC

Jaroslav Kováč (Prof, Ing, CSc) was born in Šafárikovo, Slovakia, in 1947. He graduated from the FEI STU, Bratislava, in 1970. Since 1971 he has been engaged in the research of optoelectronic devices technology at the Microelectronics Department of FEI STU. He received a CSc (PhD) degree from STU Bratislava, in 1983. Since 1991 he has been the team leader of the Optoelectronic and microwave group at the Department of Electronics and Phototonics.

Marek Tlaczala (Prof Dr Hab) was born in Bydgoszcz in 1949. He graduated in Electronics from Faculty of Electronics in 1972. In 19731976 he worked at the Electrotechnical University at Sankt Petersburg. He received his $\mathrm{PhD}$ degree in Electronic Engineering in 1976 at Electrotechnical University in Sankt Petersburg. In 2002 he received the DSc degree (habilitation). At present he is a Full Professor (from 2009) and Head of the Semiconductor Devices Lab. in Faculty of Microsystem Electronics and Photonics Wroclaw University of Technology. Vice-Director of Center of Advanced Materials and Nanotechnology at Wroclaw University of Technology. Member of Committee of Polish Vacuum Society and, at this moment he is a scientific secretary of the society. Member of: Polish Committee of Electronics and Telecommunication Polish Academy of Science, Thin Layers Division, Microelectronic Division and Electronic Materials Technology Division of Polish Academy of Sciences. His main research interest concerns crystal growth of thin and low dimensional heterostructures of AIII-BV and AIIIBV-N semiconductor compounds for optoelectronics and microelectronics application. He is a specialist in designing and construction of advanced apparatus for epitaxial crystal growth and in theoretical approach to the semiconductor materials processing technology. Many of his original works were published in scientific journals (he authored or co-authored 160 papers). During the last three years he has been a principal investigator of 11 scientific grants. 